\title{
The Cauchy Problem for the Heat Equation with a Random Right Part from the Space $\operatorname{Sub}_{\varphi}(\Omega)$
}

\author{
Yuriy Kozachenko, Anna Slyvka-Tylyshchak \\ Department of Probability Theory, Statistics and Actuarial Mathematics, The Faculty of Mechanics and \\ Mathematics, Taras Shevchenko National University of Kyiv, Kyiv, Ukraine \\ Email: yvk@univ.kiev.ua, aslyvka@ukr.net
}

Received 16 May 2014; revised 24 June 2014; accepted 7 July 2014

Copyright @ 2014 by authors and Scientific Research Publishing Inc.

This work is licensed under the Creative Commons Attribution International License (CC BY). http://creativecommons.org/licenses/by/4.0/

\section{(c) (i) Open Access}

\begin{abstract}
The influence of random factors should often be taken into account in solving problems of mathematical physics. The heat equation with random factors is a classical problem of the parabolic type of mathematical physics. In this paper, the heat equation with random right side is examined. In particular, we give conditions of existence with probability, one classical solutions in the case when the right side is a random field, sample continuous with probability one from the space $\operatorname{Sub}_{\varphi}(\Omega)$. Estimation for the distribution of the supremum of solutions of such equations is founded.
\end{abstract}

\section{Keywords}

\section{Cauchy Problem, Heat Equation, Stochastic Process}

\section{Introduction}

The subject of this work is at the intersection of two branches of mathematics: mathematical physics and stochastic processes.

The physical formulation of problems of mathematical physics with random factors was studied by Kampe de Feriet [1]. In the works [2] and [3], a new approach studying the solutions of partial differential equations with random initial conditions was proposed. The authors investigate the convergence in probability of the sequence of function spaces of partial sums approximating the solution of a problem. The mentioned approach was used in the works [4]-[7]. In the paper [3], the application of the Fourier method for the homogeneous 
hyperbolic equation with Gaussian initial conditions is justified. The conditions of the existence of the classical solution of this equation in terms of correlation functions are also studied. Homogeneous hyperbolic equation with random initial conditions from the space $\operatorname{Sub}_{\varphi}(\Omega)$ is considered in [8]-[11]. The model of a solution of a hyperbolic type equation with random initial conditions was investigated in the papers [12] [13]. There is a study on a boundary-value problem of mathematical physics for the inhomogeneous hyperbolic equation with $\varphi$-subgaussian in right part [8] [14]. The parabolic type equations of Mathematical Physics with random factors of Orlicz spaces have been studied in the papers [15] [16]. Further references can be found in [8] [17]-[21].

We consider a Cauchy problem for the heat equations with a random right part. We study the inhomogeneous heat equation on a line with a random right part. We consider the right part as a random function of the space $\operatorname{Sub}_{\varphi}(\Omega)$. The Gaussian stochastic process with zero mean belongs to $\operatorname{Sub}_{\varphi}(\Omega)$ [22]. The conditions of existence with probability one of the classical solution of this problem are investigated. For such a problem has been got the estimation for the distribution of the supremum solution.

The paper consists of the introduction and three parts. Section 2 contains necessary definitions and results of the theory of the $\operatorname{Sub}_{\varphi}(\Omega)$ space. In Section 3, we consider heat equations with random right-hand side. For such problem conditions of existence, with probability one, of classical solution with random right-hand side from the space $L_{p}(\Omega)$ are found. The estimation for distribution of supremum of this problem has been got in Section 4.

\section{Random Processes from $\operatorname{Sub}_{\varphi}(\Omega)$ Space}

Definition 1. [23] An even continuous convex function $u(x), x \in R^{1}$ such that $u(0)=0$ and $u(x)>0$ for $x \neq 0$ and $\lim _{x \rightarrow 0} \frac{u(x)}{x}=0, \lim _{x \rightarrow \infty} \frac{u(x)}{x}=\infty$ is called an $N$-function.

Definition 2. [21] We say an $N$-function $u$ satisfies the $q$-condition if there exist constants $z_{0}>0$, $k>0, \quad A>0$ such that $u(x) u(y) \leq A u(k x y)$ for all $x>z_{0}, \quad y>z_{0}$.

Lemma 1. [21] Let $u(x)$ be an $N$-function. Then

1. $u(\alpha x) \leq \alpha u(x)$ for $0 \leq \alpha \leq 1$ and $x \in R$;

2. $u(\alpha x) \geq \alpha u(x)$ for $\alpha>1$ and $x \in R$;

3. $u(|x|+|y|) \leq u(x)+u(y)$ for $x, y \in R$;

4. The function $u(x) / x$ is non decreasing for $x>0$.

Lemma 2. [21] Let $u^{(-1)}(x)$ be the inverse to an $N$-function $u(x)$ for $x>0$. Then $u^{(-1)}(x)$ is a convex increasing function such that

1. $u^{(-1)}(\alpha x) \leq \alpha u^{(-1)}(x)$ for $0 \leq \alpha \leq 1$ and $x \in R$;

2. $u^{(-1)}(\alpha x) \geq \alpha u^{(-1)}(x)$ for $\alpha>1$ and $x \in R$;

3. $u^{(-1)}(|x|+|y|) \leq u^{(-1)}(x)+u^{(-1)}(y)$ for $x, y \in R$;

4. the function $u(x) / x$ is nonincreasing for $x>0$.

Definition 3. [23] Let $u(x)$ be an $N$-function. The function $u^{*}(x)=\sup _{y \in R}(x y-u(y))$ is called the Young-

Fenchel transform of the function $u(x)$. The function $u^{*}(x)$ is an $N$-function as well.

Let $\{\Omega, \mathfrak{I}, P\}$ be a standard probability space.

Definition 4. [21] Let $\varphi(x)$ be an $N$-function for which there exist constants $x_{0}>0$ and $c>0$ such that $\varphi(x)=c x^{2}$ for $|x|<x_{0}$. The set of random variables $\xi(\varpi), \varpi \in R$, is called the space $\operatorname{Sub}_{\varphi}(\Omega)$ generated by the $N$-function $\varphi(x)$ if $E \xi=0$ and there exists a constant $a_{\xi}$ such that

$E \exp \{\lambda \xi\} \leq \exp \left\{\varphi\left(\lambda a_{\xi}\right)\right\}$ for all $\lambda \in R^{1}$.

The space $\operatorname{Sub}_{\varphi}(\Omega)$ is a Banach space with respect to the norm [21] $\tau_{\varphi}=\sup _{\lambda \neq 0} \frac{\varphi^{(-1)}(\ln E \exp \{\lambda \xi\})}{|\lambda|}$. 
Definition 5. [23] The stochastic process $X=\{X(t), t T\}$ belongs to space $\operatorname{Sub}_{\varphi}(\Omega),\left(X \in \operatorname{Sub}_{\varphi}(\Omega)\right)$ if $X(t) \in \operatorname{Sub}_{\varphi}(\Omega)$ for all $t \in T$.

Remark 1. [24] The Gaussian stochastic process $X(t)$ with zero mean belongs to $\operatorname{Sub}_{\varphi}(\Omega)$, where $\phi(x)=x^{2} / 2$ and $\tau(X(t))=\left(E(X(t))^{2}\right)^{1 / 2}$.

\section{A Family of Strongly $\operatorname{Sub}_{\varphi}(\Omega)$ Random Variables and a Family Strongly $\operatorname{Sub}_{\varphi}(\Omega)$ Stochastic Processes}

Lemma 3. [21] If $\xi \in \operatorname{Sub}_{\varphi}(\Omega)$, then there exists a constant $C>0$ such that $\left(E(\xi)^{2}\right)^{1 / 2} \leq C \tau_{\phi}(\xi)$.

Definition 6. [21] The random variable $\xi \in \operatorname{Sub}_{\varphi}(\Omega)$ is called strongly $\operatorname{Sub}_{\varphi}(\Omega)$, $\left(\operatorname{SSub}_{\varphi}(\Omega)\right)$ random variable if $\tau_{\phi}(\xi)=\left(E \xi^{2}\right)^{1 / 2}$.

Properties and applications of $\operatorname{SSub}_{\varphi}(\Omega)$ random variables and stochastic processes from $\operatorname{SSub}_{\varphi}(\Omega)$ can be found in [21].

Definition 7. [7] A family $\Delta$ of random variables $\xi$ of the space $\operatorname{Sub}_{\varphi}(\Omega)$ is called $\operatorname{SSub}_{\varphi}(\Omega)$ family if

$$
\tau_{\varphi}\left(\sum_{i \in I} \lambda_{i} \xi_{i}\right)=\left(E\left(\sum_{i \in I} \lambda_{i} \xi_{i}\right)^{2}\right)^{1 / 2}
$$

for all $\lambda_{i} \in R^{1}$, where $I$ is at most countable and $\xi_{i} \in \Delta_{i}, i \in I$.

Theorem 1. [7] Let $\Delta$ be a strongly $\operatorname{Sub}_{\varphi}(\Omega)$ family of random variables. Then the linear closure $\bar{\Delta}$ of the family $\Delta$ in the space $L_{2}(\Omega)$ and in the mean square sense is a strongly $\operatorname{Sub}_{\varphi}(\Omega)$ family.

Definition 8. [21] The stochastic process $X_{i}=\left\{X_{i}(t), t \in T, i \in I\right\}$ is called an $\operatorname{SSub}_{\varphi}(\Omega)$ process if the family of random variables $X_{i}=\left\{X_{i}(t), t \in T, i \in I\right\}$ is an $\operatorname{SSub}_{\varphi}(\Omega)$.

Theorem 2. [7] Let $X_{i}=\left\{X_{i}(t), t \in T, i \in I\right\}$ be a family of jointly strongly $\operatorname{Sub}_{\varphi}(\Omega)$ stochastic processes. Then $(T, \theta, \mu)$ is a measurable space. If $\left\{\phi_{k_{i}}(t) i \in I, k=\overline{1, \infty}\right\}$ is a family of measurable functions in $(T, \theta, \mu)$ and the integral $\xi_{k_{i}}=\int_{T} \phi_{k}(t) X_{i}(t) \mathrm{d} \mu(t)$ is well defined in the mean square sense, than the family of random variables $\Delta_{\xi}=\left\{\xi_{k_{i}}, i \in I, k=\overline{1, \infty}\right\}$ is an $\operatorname{Sub}_{\varphi}(\Omega)$ family.

Theorem 3. [9] Let $R^{k}$ be the $k$-dimensional space,

$\mathrm{d}(t, s)=\max _{1 \leq i \leq k}\left|t_{i}-s_{i}\right|, \quad T=\left\{0 \leq t_{i} \leq T_{i}, i=1,2, \cdots, k\right\}, T_{i}>0 . \quad X_{n}=\left\{X_{n}(t), t \in T\right\} \in \operatorname{Sub}_{\varphi}(\Omega)$. Assume that the process $X_{n}(t)$ is separable and

$\sup _{\mathrm{d}(t, s) \leq h} \tau_{\phi}\left(X_{n}(t)-X_{n}(s)\right) \leq \sigma(h)$ where $\sigma(h)$ is a monotone increasing continuous function such that $\sigma(h) \rightarrow 0$ as $h \rightarrow 0$. We also assume that $\int_{0+} \psi\left(\ln \frac{1}{\sigma^{(-1)}(\varepsilon)}\right) \mathrm{d} \varepsilon<\infty$, where $\psi(u)=\frac{u}{\phi^{(-1)}(u)}$ and $\sigma^{(-1)}(\varepsilon)$ is the inverse function to $\sigma(\varepsilon)$. If the processes $X_{n}(t)$ converge in probability to the process $X(t)$ for all $t \in T$, then $X_{n}(t)$ converge in probability in the space $C(T)$.

Theorem 4. [9] Let $T=\left\{a_{i} \leq x_{i} \leq b_{i}, i=1, \cdots, m\right\}$ and let $\xi(X), X \in T$, be a separable random field such that $\xi(X) \in \operatorname{SSub}_{\varphi}(\Omega)$. Put $B_{0000}(X, Y)=E \xi(X) \xi(Y)$ and assume that the partial derivatives

$$
B_{i 0 i 0}(X, Y)=\frac{\partial^{2} B(X, Y)}{\partial x_{i} \partial y_{i}}, i=1, \cdots, m \text { and }
$$




$$
B_{i k i k}(X, Y)=\frac{\partial^{4} B(X, Y)}{\partial x_{i} \partial y_{i} \partial x_{k} \partial y_{k}}, \quad i=1, \cdots, m, \quad k=1, \cdots, m
$$

exist. Let there exist a monotone increasing continuous function $\sigma_{z}(h)>0, h>0$, such that $\sigma_{z}(h) \rightarrow 0$ as $h \rightarrow 0$ for $z=(0,0,0,0), \quad z=(i, 0, i, 0), \quad i=1, \cdots, m$ and $z=(i, k, i, k), i, k=1, \cdots, m$. Assume that

$$
\sup _{\substack{\left|x_{i}-y_{i}\right| \leq h \\ i=1, \cdots, m}}\left(B_{z}(X, X)+B_{z}(Y, Y)-2 B_{z}(X, Y)\right)^{1 / 2} \leq \sigma_{z}(h)
$$

If $\int_{0}^{\varepsilon} \psi\left(\ln \left(\frac{1}{\phi^{(-1)}(u)}\right)\right) \mathrm{d} u<\infty$ for all $z$ and for sufficiently small $\varepsilon>0$ where $\psi(u)=\frac{u}{\phi^{(-1)}(u)}$, then with probability one the partial derivatives $\frac{\partial \xi(X)}{\partial x_{i}}, \frac{\partial^{2} \xi(X)}{\partial x_{i} \partial x_{j}}, i, j=1, \cdots, m$, exist and are continuous.

\section{The Heat Equations with Random Right Part}

We consider the Cauchy problem for the heat equation

$$
\frac{\partial u(x, t)}{\partial t}=a^{2} \frac{\partial^{2} u(x, t)}{\partial x^{2}}+\xi(x, t),
$$

$-\infty<x<+\infty, t>0$, subject to the initial condition

$$
u(x, 0)=0,-\infty<x<+\infty .
$$

Let the function $\xi(x, t)=\{\xi(x, t), x R, t>0\}$ is a random field sample continuity with probability one from the space $\operatorname{Sub}_{\varphi}(\Omega)$, such that $E \xi(x, t)=0, E(\xi(x, t))^{2}<+\infty$. Let us denote $B(x, t, z, s)=E \xi(x, t) \xi(z, s)$. Let $B(x, t, z, s)$ be a continuous function. Problem when the function $\xi(x, t)$ nonrandom has been seen in [25].

Lemma 4. Let $\xi(x, t)$ is a random field, sample continuity for each $t>0$ with probability one, there is a continuous derivative $\frac{\partial \xi(x, t)}{\partial x}$ for $x \in R$ and satisfy condition

$$
\int_{R} \sqrt{E\left(\xi^{2}(x, t)\right)} \mathrm{d} x<\infty .
$$

Then for the function $\xi(x, t)$ for each $t>0$ the integral Fourier transform

$$
\tilde{\xi}(y, \tau)=\frac{1}{\sqrt{2 \pi}} \int_{-\infty}^{+\infty} \cos y x \xi(x, \tau) \mathrm{d} x
$$

exist and $\xi(x, \tau)=\frac{1}{\sqrt{2 \pi}} \int_{-\infty}^{+\infty} \cos y x \tilde{\xi}(x, \tau) \mathrm{d} y$.

Proof. Since, by Fubini's theorem, $\int_{R} E|\xi(x, t)| \mathrm{d} x<\int_{R} \sqrt{E\left(\xi^{2}(x, t)\right)}$, we deduce that the integral $\int_{R}|\xi(x, t)| \mathrm{d} x<\infty$ exist with probability one, and therefore the integral $\int_{-\infty}^{+\infty} \cos y x \xi(x, \tau) \mathrm{d} x$, and therefore it implies from [26] that the integral Fourier transform

$$
\tilde{\xi}(y, \tau)=\frac{1}{\sqrt{2 \pi}} \int_{-\infty}^{+\infty} \cos y x \xi(x, \tau) \mathrm{d} x
$$

exist, and the inverse integral Fourier transform 


$$
\xi(x, \tau)=\frac{1}{\sqrt{2 \pi}} \int_{-\infty}^{+\infty} \cos y x \tilde{\xi}(x, \tau) \mathrm{d} x
$$

exist.

Theorem 5. Let the conditions of Lemma 4 be satisfied and

$$
\begin{gathered}
G(y, t)=\frac{1}{\sqrt{2 \pi}} \int_{0}^{t} \mathrm{e}^{-a^{2} y^{2}(t-\tau)} \tilde{\xi}(y, \tau) \mathrm{d} \tau, \\
\tilde{\xi}(y, \tau)=\frac{1}{\sqrt{2 \pi}} \int_{-\infty}^{+\infty} \cos y x \xi(x, \tau) \mathrm{d} x
\end{gathered}
$$

and

$$
u(x, t)=\int_{-\infty}^{+\infty} \cos y x G(y, t) \mathrm{d} y
$$

If the following integrals exist

$$
\begin{aligned}
\int_{-\infty}^{+\infty} y \sin y x G(y, t) \mathrm{d} y & , \int_{-\infty}^{+\infty} y^{s} \cos y x G(y, t) \mathrm{d} y, \\
s= & 0,2
\end{aligned}
$$

and for all $A>0$ and $T>0$ there exists a sequence $a_{n}, a_{n} \rightarrow \infty$ for $n \rightarrow \infty$, such that the sequence of integrals

$$
\begin{gathered}
\int_{-a_{n}}^{+a_{n}} y \sin y x G(y, t) \mathrm{d} y, \\
\int_{-a_{n}}^{+a_{n}} y^{s} \cos y x G(y, t) \mathrm{d} y, \quad s=0,2 .
\end{gathered}
$$

converges in probability, uniformly for $|x| \leq A, 0 \leq t \leq T$, then $u(x, t)$ is the classical solution to the problem (1) and (2).

Proof. Since the integrals (5) and (6) converges in probability uniformly for $|x| \leq A, 0 \leq t \leq T$, there exists a subsequence $b_{n}, b_{n} \rightarrow \infty$ as $n \rightarrow \infty$, such that

$$
\int_{-b_{n}}^{+b_{n}} y \sin y x G(y, t) \mathrm{d} y, \int_{-b_{n}}^{+b_{n}} y^{s} \cos y x G(y, t) \mathrm{d} y, \quad s=0,2,
$$

converges with probability one to

$$
\int_{-\infty}^{+\infty} y \sin y x G(y, t) \mathrm{d} y, \int_{-\infty}^{+\infty} y^{s} \cos y x G(y, t) \mathrm{d} y, \quad s=0,2,
$$

uniformly for $|x| \leq A, \quad 0 \leq t \leq T$, Let

$$
u_{b_{n}}(x, t)=\int_{-b_{n}}^{+b_{n}} \cos y x G(y, t) \mathrm{d} y .
$$

By deriving (7) with respect to $x$ and $t$, we easily see that

$$
\begin{aligned}
\frac{\partial^{2} u_{b_{n}}(x, t)}{\partial t}= & -a^{2} \int_{-b_{n}}^{+b_{n}} y^{2} \cos y x G(y, t) \mathrm{d} y+\frac{1}{\sqrt{2 \pi}} \int_{-b_{n}}^{b_{n}} \cos y x \tilde{\xi}(y, \tau) \mathrm{d} y, \\
& \frac{\partial^{2} u_{b_{n}}(x, t)}{\partial x^{2}}=-\int_{-b_{n}}^{+b_{n}} y^{2} \cos y x G(y, t) \mathrm{d} y,
\end{aligned}
$$




$$
\frac{\partial u_{b_{n}}(x, t)}{\partial t}=-a^{2} \frac{\partial^{2} u_{b_{n}}(x, t)}{\partial x^{2}}+\frac{1}{\sqrt{2 \pi}} \int_{-b_{n}}^{b_{n}} \cos y x \tilde{\xi}(y, \tau) \mathrm{d} y,
$$

$t>0, x \in R$. Since for $n \rightarrow \infty \frac{\partial u_{b_{n}}(x, t)}{\partial t}$ converges to $\frac{\partial u(x, t)}{\partial t}$, and $\frac{\partial^{2} u_{b_{n}}(x, t)}{\partial x^{2}}$ converges to $\frac{\partial^{2} u(x, t)}{\partial x^{2}}$ uniformly for $|x| \leq A, \quad 0 \leq t \leq T$ with probability one, we conclude that $u(x, t)$ satisfies Equation (1).

Indeed,

$$
\frac{\partial u(x, t)}{\partial t}=-a^{2} \int_{-\infty}^{+\infty} y^{2} \cos y x G(y, t) \mathrm{d} y+\frac{1}{\sqrt{2 \pi}} \int_{-\infty}^{+\infty} \cos y x \tilde{\xi}(y, \tau) \mathrm{d} y
$$

$$
=a^{2} \frac{\partial^{2} u(x, t)}{\partial x^{2}}+\xi(x, t)
$$

Lemma 5. [9] Let $\xi(x, t)$ be a random field, sample continuity from the space $\operatorname{Sub}_{\varphi}(\Omega)$. Let $B(x, t, v, s)$ be the correlation function of the field $\xi(x, t)$. For all $t>0, s>0$ assume that:

1. The derivatives $\frac{\partial^{k} B(x, t, v, s)}{\partial x^{l} \partial v^{m}}, k=0, \cdots, 4, \quad l+m=k$ exist;

$2 \int_{-\infty}^{+\infty} \int_{-\infty}^{+\infty} \frac{\partial^{k} B(x, t, v, s)}{\partial x^{l} \partial v^{m}} \mathrm{~d} x \mathrm{~d} v \leq B(k, l, m)<\infty, k=0, \cdots, 4, \quad l+m=k$

3. $\frac{\partial^{k} B(x, t, v, s)}{\partial x^{l} \partial v^{m}} \rightarrow 0, k=0, \cdots, 4, \quad l+m=k$, at $x \rightarrow \infty$ or $v \rightarrow \infty$.

Then Lebesgue integrals

$$
\int_{-\infty}^{\infty} y \sin y x G(y, t) \mathrm{d} y, \quad \int_{-\infty}^{+\infty} y^{s} \cos y x G(y, t) \mathrm{d} y, \quad s=0,2
$$

exist with probability one.

Proof. We shall prove the existence of the integral

$$
\int_{-\infty}^{+\infty} y^{2} \cos y x G(y, t) \mathrm{d} y
$$

For existence of this integral with probability one it is enough to prove that there exists following integral

$$
\int_{-\infty}^{+\infty} y^{2} E|G(y, t)| \mathrm{d} y .
$$

There is an inequality

$$
\int_{-\infty}^{+\infty} y^{2} E|G(y, t)| \mathrm{d} y \leq \int_{-\infty}^{+\infty} y^{2} \sqrt{E(G(y, t))^{2}} \mathrm{~d} y
$$

Consider

$$
\begin{aligned}
& E(G(y, t))^{2} \\
& =\frac{1}{2 \pi} \int_{0}^{t} \int_{0}^{t} \mathrm{e}^{-a^{2} y^{2}(t-\tau)} \mathrm{e}^{-a^{2} y^{2}(t-s)} E(\tilde{\xi}(y, \tau) \tilde{\xi}(y, s)) \mathrm{d} \tau \mathrm{d} s \\
& =E(\tilde{\xi}(y, \tau) \tilde{\xi}(y, s))=\frac{1}{2 \pi} \int_{-\infty}^{+\infty} \int_{-\infty}^{+\infty} \cos y x \cos y v \cdot \xi(x, \tau) \xi(v, s) \mathrm{d} x \mathrm{~d} v \\
& =\frac{1}{2 \pi} \int_{-\infty}^{+\infty} \int_{-\infty}^{+\infty} \cos y x \cos y v B(x, \tau, v, s) \mathrm{d} x \mathrm{~d} v .
\end{aligned}
$$

Integrating by parts and using the conditions of the lemma, we obtain for $y \neq 0$ 


$$
\begin{aligned}
E(\tilde{\xi}(y, \tau) \tilde{\xi}(y, s))= & \frac{1}{2 \pi} \int_{-\infty}^{+\infty} \int_{-\infty}^{+\infty} \frac{\cos y x \cos y v}{y^{4}} \cdot \frac{\partial^{4} B(x, t, v, s)}{\partial x^{2} \partial v^{s}} \mathrm{~d} x \mathrm{~d} v \\
|E(\tilde{\xi}(y, \tau) \tilde{\xi}(y, s))| & =\frac{1}{2 \pi} \int_{-\infty}^{+\infty} \int_{-\infty}^{+\infty} \frac{1}{y^{4}} \cdot\left|\frac{\partial^{4} B(x, t, v, s)}{\partial x^{2} \partial v^{s}}\right| \mathrm{d} x \mathrm{~d} v \\
& \leq \frac{1}{2 \pi} \cdot \frac{1}{y^{4}} \cdot B(4,2,2)
\end{aligned}
$$

Then

$$
\begin{aligned}
& (E(G(y, t)))^{2} \\
& =\left(\frac{1}{2 \pi}\right)^{2} \frac{1}{y^{4}} B(4,2,2) \int_{0}^{t} \int_{0}^{t} \mathrm{e}^{-a^{2} y^{2}(t-\tau)} \mathrm{e}^{-a^{2} y^{2}(t-\tau)} \mathrm{d} \tau \mathrm{d} s \\
& =\left(\frac{1}{2 \pi}\right)^{2} \cdot \frac{1}{a^{4} y^{8}} B(4,2,2)\left(1-\mathrm{e}^{-a^{2} y^{2} t}\right)^{2} .
\end{aligned}
$$

Therefore

$$
\begin{aligned}
& \int_{-\infty}^{+\infty} y^{2} \sqrt{E(G(y, t))^{2}} \mathrm{~d} y \\
& \leq \frac{\sqrt{B(4,2,2)}}{2 \pi a^{2}} \int_{-\infty}^{+\infty} \frac{\left(1-\mathrm{e}^{-a^{2} y^{2} t}\right)}{y^{2}} \mathrm{~d} y
\end{aligned}
$$

for $y \neq 0$. The latter integral converges under $y \in R$. The existence of integrals $\int_{-\infty}^{+\infty} y \sin y x G(y, t) \mathrm{d} y$, $\int_{-\infty}^{+\infty} \cos y x G(y, t) \mathrm{d} y \quad$ can be proved similarly.

Lemma 6. [15] Let a function $X(\lambda, u), \lambda>0$ and $u>0$ be such that:

1. $\sup _{\substack{0 \leq u<+\infty \\ 0 \leq \lambda<+\infty}}|X(\lambda, u)| \leq B$

2. $|X(\lambda, u)-X(\lambda, v)| \leq C \lambda|u-v|$ for all $u>0, \quad v>0$. Let $\varphi(\lambda), \lambda>0$ be a continuous increasing function such that $\varphi(\lambda)>0$ for all $\lambda>0$, and the function $\lambda / \varphi(\lambda)$ is increasing for $\lambda>v_{0}$, and for some constant $v_{0}>0$.

$$
\text { Then }|X(\lambda, u)-X(\lambda, v)| \leq \max (C, 2 B) \frac{\varphi\left(\lambda+v_{0}\right)}{\varphi\left(\frac{1}{|u-v|}+v_{0}\right)}
$$

for all $\lambda \geq 0$ and $v>0$.

Corollary 1. Let in the conditions of Lemma 6 the function $\varphi(\lambda)=(\ln (1+\lambda))^{\delta}, \lambda>0, \delta>0$. Then

$$
|X(\lambda, u)-X(\lambda, v)| \leq \max (C, 2 B) \frac{\left(\ln \left(\lambda+\mathrm{e}^{\delta}\right)\right)^{\delta}}{\left(\ln \left(\frac{1}{|u-v|}+\mathrm{e}^{\delta}\right)\right)^{\delta}}
$$

for all $\delta>0$.

Proof. Indeed, it is easy to show that the function

$\lambda / \varphi(\lambda)$ increases with $v_{0}=\mathrm{e}^{\delta}-1$. Therefore in Lemma 6 taking function $\varphi(\lambda)=(\ln (1+\lambda))^{\delta} \quad \lambda>0$, $\delta>0$, we obtain the inequality 8 . 


\section{Corollary 2.}

$$
\begin{gathered}
\left|\mathrm{e}^{-a^{2} y^{2}\left(t-t_{1}\right)}-1\right| \leq \max \left(1, a^{2}\right) \frac{\left(\ln \left(y^{2}+\mathrm{e}^{\delta}\right)\right)^{\delta}}{\left(\ln \left(\frac{1}{\left|t-t_{1}\right|}+\mathrm{e}^{\delta}\right)\right)^{\delta}} . \\
\left|\cos y x-\cos y x_{1}\right| \leq \frac{\left(\ln \left(y^{2}+\mathrm{e}^{\delta}\right)\right)^{\delta}}{\left(\ln \left(\frac{1}{\left|x-x_{1}\right|}+\mathrm{e}^{\delta}\right)\right)^{\delta}}
\end{gathered}
$$

for some $\delta>0$.

Remark 2. If in the conditions of Corollary $2\left|t-t_{1}\right| \leq h,\left|x-x_{1}\right| \leq h$, then for sufficiently small $h$ inequality (9) and (10) will have the form

$$
\begin{gathered}
\left|\mathrm{e}^{-a^{2} y^{2}\left(t-t_{1}\right)}-1\right| \leq \max \left(1, a^{2}\right) \frac{\left(\ln \left(y^{2}+\mathrm{e}^{\delta}\right)\right)^{\delta}}{\left|\ln \left(|h|+\mathrm{e}^{\delta}\right)\right|^{\delta}} . \\
\left|\cos y x-\cos y x_{1}\right| \leq \frac{\left(\ln \left(y+\mathrm{e}^{\delta}\right)\right)^{\delta}}{\left|\ln \left(|h|+\mathrm{e}^{\delta}\right)\right|^{\delta}} .
\end{gathered}
$$

Let $u_{a_{n}}^{(0)}(x, t)=\int_{-a_{n}}^{+a_{n}} \cos y x G(y, t) \mathrm{d} y, \quad u_{a_{n}}^{(1)}(x, t)=\int_{-a_{n}}^{+a_{n}} y \sin y x G(y, t) \mathrm{d} y, \quad u_{a_{n}}^{(2)}(x, t)=\int_{-a_{n}}^{+a_{n}} y^{2} \cos y x G(y, t) \mathrm{d} y$,

Theorem 6. Let $\xi(x, t)$ be a random field, sample continuous with probability one from the $\operatorname{Sub}_{\varphi}(\Omega)$ and the conditions of Lemma 4 and Lemma 5 hold,

$$
\sup _{\substack{x<x_{1}\left|\leq h, t-t_{1}\right| \leq h}} \tau_{\varphi}\left(u_{a_{n}}^{(k)}(x, t)-u_{a_{n}}^{(k)}\left(x_{1}, t_{1}\right)\right) \leq \sigma_{k}(h),
$$

For $k=0,1,2$, where $\sigma_{k}(h)$ is a monotone increasing continuous function such that $\sigma_{k}(h) \rightarrow 0$ as $h \rightarrow 0$, moreover,

$$
\int_{0+} \psi\left(\ln \frac{1}{\sigma_{k}^{(-1)}(\varepsilon)}\right) \mathrm{d} \varepsilon<\infty,
$$

where $\psi(u)=\frac{u}{\phi^{(-1)}(u)}$, and $\sigma_{k}^{(-1)}(\varepsilon)$ is the inverse function to $\sigma_{k}(\varepsilon)$. Then the function $u(x, t)$ which is represented in the form (4) is a classical solution to the problems (1) and (2).

Proof. This theorem follows from Theorems 5 and 3.

Example 1. Let $\varphi(x)$ be a function such that $\varphi(x)=|x|^{p}$, for some $p>1$ and all $|x|>1$. Then $\psi(x)=x^{1-\frac{1}{p}}$ for $x>1$ and condition (11) holds for all $\varepsilon>0$

$$
\int_{0+}\left(\ln \frac{1}{\sigma_{k}^{(-1)}(\varepsilon)}\right)^{1-\frac{1}{p}} \mathrm{~d} \varepsilon<\infty
$$

Condition (12) holds if $\sigma_{k}(h)=\frac{C_{k}}{\left.|\ln | h\right|^{\delta}}$, for $\delta>1-1 / p$, 
$C_{k}>0, k=0,1,2$. In this case, the condition of Theorem 6 is satisfied if for $k=0,1,2$ there exist constants $C_{k}>0$ such that

$$
\left(E\left|u_{a_{n}}^{(k)}(x, t)-u_{a_{n}}^{(k)}\left(x_{1}, t_{1}\right)\right|^{2}\right)^{1 / 2} \leq \frac{C_{k}}{\left.|\ln | h\right|^{\delta}},
$$

For $\delta>1-1 / p$ all $n=1,2, \cdots$, and sufficiently small $|h|$.

Theorem 7. Let $\xi(x, t)$ be a random field, sample continuous with probability one from the space $\operatorname{SSub}_{\varphi}(\Omega)$, where $\varphi(x)$ is a function such that $\phi(x)=|x|^{p}$ for some $p>1$ and all $|x|>1$ and the conditions of Lemma 4 and Lemma 5 hold and $\int_{-\infty}^{+\infty}\left(E|\xi(x, \tau)|^{2}\right)^{1 / 2} \mathrm{~d} x<\theta \int_{-\infty}^{+\infty}\left(E\left|\frac{\partial \xi(x, \tau)}{\partial x}\right|^{2}\right)^{1 / 2} \mathrm{~d} x<\theta_{1}$, $\int_{-\infty}^{+\infty}\left(E\left|\frac{\partial^{2} \xi(x, \tau)}{\partial x^{2}}\right|^{2}\right)^{1 / 2} \mathrm{~d} x<\theta_{2}$ for some $\theta>0, \theta_{1}>0, \theta_{2}>0$. Then the function $u(x, t)$ which is represented in the form (4) is classical solution to the problems (1) and (2).

Proof. It follows from Lemma 5 that there exist integrals with probability one

$$
\int_{-\infty}^{+\infty} y \sin y x G(y, t) \mathrm{d} y, \int_{-\infty}^{+\infty} y^{s} \cos y x G(y, t) \mathrm{d} y, \quad s=0,2 .
$$

According to Theorem 5 to make the function $u(x, t)$ be the solution of problems (1) and (2) it is sufficient to prove that integrals (5) and (6) converge uniformly in probability in $|x| \leq A, 0 \leq t \leq T$ to the integrals

$$
\int_{-\infty}^{+\infty} y \sin y x G(y, t) \mathrm{d} y, \int_{-\infty}^{+\infty} y^{s} \cos y x G(y, t) \mathrm{d} y, \quad s=0,2,
$$

for any $A>0, T>0$. According to Theorem 6, using the Example (1), to make integral (5) and (6) converge in probability in $C(\tilde{T})$ the following conditions must hold

$$
\begin{aligned}
& \left(E\left|u_{a_{n}}^{(k)}(x, t)-u_{a_{n}}^{(k)}\left(x_{1}, t_{1}\right)\right|^{2}\right)^{1 / 2} \leq \frac{C_{k}}{|\ln | h||^{\delta}}, \\
& k=0,1,2 .
\end{aligned}
$$

Using generalized Minkovskoho inequality we obtain

$$
\begin{aligned}
& \left(E\left|u_{a_{n}}^{(0)}(x, t)-u_{a_{n}}^{(0)}\left(x_{1}, t_{1}\right)\right|^{2}\right)^{1 / 2} \\
& =\left(\left.E\left|\int_{-a_{n}}^{a_{n}} \cos y x G(y, t) \mathrm{d} y-\int_{-a_{n}}^{a_{n}} \cos y x_{1} G\left(y, t_{1}\right) \mathrm{d} y\right|^{2}\right|^{1 / 2}\right. \\
& =\left(\left.E\left|\int_{-a_{n}}^{a_{n}}\left[\cos y x G(y, t)-\cos y x_{1} G\left(y, t_{1}\right)\right] \mathrm{d} y\right|^{2}\right|^{1 / 2}\right. \\
& =\left(E\left[\left.\int_{-a_{n}}^{a_{n}}\left[\left(\cos y x-\cos y x_{1}\right) G\left(y, t_{1}\right)+\left(G(y, t)-G\left(y, t_{1}\right)\right) \cos y x\right] \mathrm{d} y\right|^{2}\right)^{1 / 2}\right. \\
& \leq \int_{-\infty}^{+\infty}\left[\left|\cos y x-\cos y x_{1}\right|\left(E\left|G\left(y, t_{1}\right)\right|^{2}\right)^{1 / 2}+\left(E\left|G(y, t)-G\left(y, t_{1}\right)\right|^{2}\right)^{1 / 2}\right] \mathrm{d} y
\end{aligned}
$$

Let $\left|x-x_{1}\right| \leq h$ and for sufficiently small $|h|$, using the inequality (10), we have 


$$
\left|\cos y x-\cos y x_{1}\right| \leq \frac{\left(\ln \left(y+\mathrm{e}^{\delta}\right)\right)^{\delta}}{\left|\ln \left(|h|+\mathrm{e}^{\delta}\right)\right|^{\delta}} .
$$

Consider

$$
\begin{aligned}
& \left(\left|G\left(y, t_{1}\right)\right|^{2}\right)^{1 / 2}=\frac{1}{\sqrt{2 \pi}} \int_{0}^{t_{1}} \mathrm{e}^{-a^{2} y^{2}(t-\tau)}\left(E|\tilde{\xi}(y, \tau)|^{2}\right)^{1 / 2} \mathrm{~d} \tau . \\
& \left(E|\tilde{\xi}(y, \tau)|^{2}\right)^{1 / 2}=\frac{1}{\sqrt{2 \pi}}\left(E\left|\int_{-\infty}^{+\infty} \cos y x \xi(x, \tau) \mathrm{d} x\right|^{2}\right)^{1 / 2} \\
& \text { It follows from Lemma } 4 \text { that } \quad \leq \frac{1}{\sqrt{2 \pi}} \int_{-\infty}^{+\infty}\left(E|\xi(x, \tau)|^{2}\right)^{1 / 2} \mathrm{~d} x \\
& <\frac{1}{\sqrt{2 \pi}} \theta \text {. }
\end{aligned}
$$

Therefore

$$
\left(E\left|G\left(y, t_{1}\right)\right|^{2}\right)^{1 / 2} \leq \frac{1}{2 \pi} \int_{0}^{t_{1}} \theta \mathrm{e}^{-a^{2} y^{2}\left(t_{1}-\tau\right)} \mathrm{d} \tau \leq \frac{\theta}{2 \pi} \cdot \frac{1}{a^{2} y^{2}}\left|1-\mathrm{e}^{-a^{2} y^{2} t_{1}}\right| .
$$

Let $t_{1}<t$ then

$$
\begin{aligned}
& \left(E\left|G(y, t)-G\left(y, t_{1}\right)\right|^{2}\right)^{1 / 2} \\
& =\frac{1}{\sqrt{2 \pi}}\left(E\left|\int_{0}^{t} \mathrm{e}^{-a^{2} y^{2}(t-\tau)} \tilde{\xi}(y, \tau) \mathrm{d} \tau-\int_{0}^{t_{1}} \mathrm{e}^{-a^{2} y^{2}\left(t_{1}-\tau\right)} \tilde{\xi}(y, \tau) \mathrm{d} \tau\right|^{2}\right)^{1 / 2} \\
& =\frac{1}{\sqrt{2 \pi}}\left(\left.E\left|\int_{0}^{t_{1}}\left[\mathrm{e}^{-a^{2} y^{2}(t-\tau)} \mathrm{e}^{-a^{2} y^{2}(t-\tau)}-\mathrm{e}^{-a^{2} y^{2}\left(t_{1}-\tau\right)}\right] \tilde{\xi}(y, \tau) \mathrm{d} \tau+\int_{t_{1}}^{t} \mathrm{e}^{-a^{2} y^{2}(t-\tau)} \tilde{\xi}(y, \tau) \mathrm{d} \tau\right|^{2}\right|^{1 / 2}\right. \\
& \leq \frac{1}{\sqrt{2 \pi}}\left(\int_{0}^{t_{1}}\left[\left|\mathrm{e}^{-a^{2} y^{2}(t-\tau)}-\mathrm{e}^{-a^{2} y^{2}\left(t_{1}-\tau\right)}\right|\left(E|\tilde{\xi}(y, \tau)|^{2}\right)^{1 / 2}\right] \mathrm{d} \tau+\int_{t_{1}}^{t} \mathrm{e}^{-a^{2} y^{2}(t-\tau)}\left(E|\tilde{\xi}(y, \tau)|^{2}\right)^{1 / 2} \mathrm{~d} \tau\right) .
\end{aligned}
$$

Let $\left|t-t_{1}\right| \leq h$ and for sufficiently small $|h|$, using the inequality (9), we have

$$
\left|\mathrm{e}^{-a^{2} y^{2}(t-\tau)}-\mathrm{e}^{-a^{2} y^{2}\left(t_{1}-\tau\right)}\right|=\mathrm{e}^{-a^{2} y^{2}\left(t_{1}-\tau\right)}\left|\mathrm{e}^{-a^{2} y^{2}\left(t_{1}-\tau\right)}-1\right| \leq \mathrm{e}^{-a^{2} y^{2}\left(t_{1}-\tau\right)} \max \left(1, a^{2}\right) \frac{\left(\ln \left(y^{2}+\mathrm{e}^{\delta}\right)\right)^{\delta}}{|\ln (h)|^{\delta}} .
$$

Therefore

$$
\begin{aligned}
& \left(E\left|G(y, t)-G\left(y, t_{1}\right)\right|^{2}\right)^{1 / 2} \\
& \leq \frac{1}{2 \pi}\left(\int_{0}^{t} \mathrm{e}^{-a^{2} y^{2}\left(t_{1}-\tau\right)} \max \left(1, a^{2}\right) \frac{\left(\ln \left(y+\mathrm{e}^{\delta}\right)\right)^{\delta}}{|\ln | h||^{\delta}} \theta \mathrm{d} \tau+\int_{t_{1}}^{t} \mathrm{e}^{-a^{2} y^{2}(t-\tau)} \theta \mathrm{d} \tau\right) \\
& =\frac{\theta}{2 \pi}\left(\max \left(1, a^{2}\right) \frac{\left(\ln \left(y^{2}+\mathrm{e}^{\delta}\right)\right)^{\delta}}{\left.|\ln | h\right|^{\delta}} \cdot \frac{1}{a^{2} y^{2}}\left|1-\mathrm{e}^{-a^{2} y^{2} t_{1}}\right|+\int_{t_{1}}^{t} \mathrm{e}^{-a^{2} y^{2}(t-\tau)} \mathrm{d} \tau\right) .
\end{aligned}
$$

Thus we obtain from (14), (15), (16) and (17) that 


$$
\begin{aligned}
& \left(E\left|u_{a_{n}}^{(0)}(x, t)-u_{a_{n}}^{(0)}\left(x_{1}, t_{1}\right)\right|^{2}\right)^{1 / 2} \\
& \leq \frac{\theta}{2 \pi} \int_{-\infty}^{+\infty}\left[\frac{\left(\ln \left(y+\mathrm{e}^{\delta}\right)\right)^{\delta}}{\left.|\ln | h\right|^{\delta}} \cdot \frac{1}{a^{2} y^{2}}\left|1-\mathrm{e}^{-a^{2} y^{2} t_{1}}\right|+\max \left(1, a^{2}\right) \frac{\left(\ln \left(y^{2}+\mathrm{e}^{\delta}\right)\right)^{\delta}}{|\ln | h||^{\delta}} \cdot \frac{1}{a^{2} y^{2}}\left|1-\mathrm{e}^{-a^{2} y^{2} t_{1}}\right|+\int_{t_{1}}^{t} \mathrm{e}^{-a^{2} y^{2}(t-\tau)} \mathrm{d} \tau\right] \mathrm{d} y \\
& =\frac{\theta}{\pi} \int_{0}^{+\infty}\left[\frac{\left(\ln \left(y+\mathrm{e}^{\delta}\right)\right)^{\delta}}{\left.|\ln | h\right|^{\delta}} \cdot \frac{1}{a^{2} y^{2}}\left|1-\mathrm{e}^{-a^{2} y^{2} t_{1}}\right|+\max \left(1, a^{2}\right) \frac{\left(\ln \left(y^{2}+\mathrm{e}^{\delta}\right)\right)^{\delta}}{|\ln | h||^{\delta}} \cdot \frac{1}{a^{2} y^{2}}\left|1-\mathrm{e}^{-a^{2} y^{2} t_{1}}\right|+\int_{t_{1}}^{t} \mathrm{e}^{-a^{2} y^{2}(t-\tau)} \mathrm{d} \tau\right] \mathrm{d} y \\
& =\frac{\theta}{\pi} \int_{0}^{1}\left[\frac{\left(\ln \left(y+\mathrm{e}^{\delta}\right)\right)^{\delta}}{\left.|\ln | h\right|^{\delta}} \cdot \frac{1}{a^{2} y^{2}}\left|1-\mathrm{e}^{-a^{2} y^{2} t_{1}}\right|+\max \left(1, a^{2}\right) \frac{\left(\ln \left(y^{2}+\mathrm{e}^{\delta}\right)\right)^{\delta}}{\left.|\ln | h\right|^{\delta}} \cdot \frac{1}{a^{2} y^{2}}\left|1-\mathrm{e}^{-a^{2} y^{2} t_{1}}\right|+\int_{t_{1}}^{t} \mathrm{e}^{-a^{2} y^{2}(t-\tau)} \mathrm{d} \tau\right] \mathrm{d} y \\
& +\frac{\theta}{\pi} \int_{1}^{+\infty}\left[\frac{\left(\ln \left(y+\mathrm{e}^{\delta}\right)\right)^{\delta}}{\left.|\ln | h\right|^{\delta}} \cdot \frac{1}{a^{2} y^{2}}\left|1-\mathrm{e}^{-a^{2} y^{2} t_{1}}\right|+\max \left(1, a^{2}\right) \frac{\left(\ln \left(y^{2}+\mathrm{e}^{\delta}\right)\right)^{\delta}}{\left.|\ln | h\right|^{\delta}} \cdot \frac{1}{a^{2} y^{2}}\left|1-\mathrm{e}^{-a^{2} y^{2} t_{1} \mid}\right|+\int_{t_{1}}^{t} \mathrm{e}^{-a^{2} y^{2}(t-\tau)} \mathrm{d} \tau\right] \mathrm{d} y \\
& =\frac{\theta}{\pi}\left(I_{1}+I_{2}\right) .
\end{aligned}
$$

Consider

$$
\begin{aligned}
I_{1} & =\int_{0}^{1}\left[\frac{\left(\ln \left(y+\mathrm{e}^{\delta}\right)\right)^{\delta}}{\left.|\ln | h\right|^{\delta}} \cdot \frac{1}{a^{2} y^{2}}\left|1-\mathrm{e}^{-a^{2} y^{2} t_{1}}\right|+\max \left(1, a^{2}\right) \frac{\left(\ln \left(y^{2}+\mathrm{e}^{\delta}\right)\right)^{\delta}}{\left.|\ln | h\right|^{\delta}} \cdot \frac{1}{a^{2} y^{2}}\left|1-\mathrm{e}^{-a^{2} y^{2} t_{1}}\right|+\int_{t_{1}}^{t} \mathrm{e}^{-a^{2} y^{2}(t-\tau)} \mathrm{d} \tau\right] \mathrm{d} y \\
& =\frac{1}{a^{2} \ln |h|^{\delta}} \int_{0}^{1} \frac{\left(\ln \left(y+\mathrm{e}^{\delta}\right)\right)^{\delta}}{y^{2}}\left|1-\mathrm{e}^{-a^{2} y^{2} t_{1}}\right| \mathrm{d} y+\frac{\max \left(1, a^{2}\right)}{\left.a^{2}|\ln | h\right|^{\delta}} \cdot \int_{0}^{1} \frac{\left(\ln \left(y^{2}+\mathrm{e}^{\delta}\right)\right)^{\delta}}{y^{2}}\left|1-\mathrm{e}^{-a^{2} y^{2} t_{1}}\right| \mathrm{d} y+\int_{0}^{1}\left(\int_{t_{1}}^{t} \mathrm{e}^{-a^{2} y^{2}(t-\tau)} \mathrm{d} \tau\right) \mathrm{d} y \\
& =\frac{1}{\left.a^{2}|\ln | h\right|^{\delta}} I_{11}+\frac{\max \left(1, a^{2}\right)}{\left.a^{2}|\ln | h\right|^{\delta}} I_{12}+I_{13} .
\end{aligned}
$$

Since $\left|1-\mathrm{e}^{-a^{2} y^{2} t_{1}}\right| \leq a^{2} y^{2} t_{1} \leq a^{2} y^{2} T$, we have

$$
\begin{gathered}
I_{11}=\int_{0}^{1} \frac{\left(\ln \left(y+\mathrm{e}^{\delta}\right)\right)^{\delta}}{y^{2}}\left|1-\mathrm{e}^{-a^{2} y^{2} t_{1}}\right| \mathrm{d} y \leq a^{2} T \int_{0}^{1}\left(\ln \left(y+\mathrm{e}^{\delta}\right)\right)^{\delta} \mathrm{d} y=a^{2} T C_{11} . \\
I_{12}=\int_{0}^{1} \frac{\left(\ln \left(y^{2}+\mathrm{e}^{\delta}\right)\right)^{\delta}}{y^{2}}\left|1-\mathrm{e}^{-a^{2} y^{2} t_{1}}\right| \mathrm{d} y \leq a^{2} T \int_{0}^{1}\left(\ln \left(y^{2}+\mathrm{e}^{\delta}\right)\right)^{\delta} \mathrm{d} y=a^{2} T C_{12} .
\end{gathered}
$$

Using that $\mathrm{e}^{-a^{2} y^{2}(t-\tau)} \leq 1, t-t_{1} \leq h$, then the $\delta>0$ and for sufficiently small $h$, we have

$$
I_{13}=\int_{0}^{1}\left(\int_{t_{1}}^{t} \mathrm{e}^{-a^{2} y^{2}(t-\tau)} \mathrm{d} \tau\right) \mathrm{d} y \leq \int_{0}^{1}\left(t-t_{1}\right) \mathrm{d} y \leq|h| \leq \frac{1}{|\ln h|^{\delta}} .
$$

So we have

$$
I_{1} \leq \frac{1}{\left.|\ln | h\right|^{\delta}}\left(T C_{11}+\max \left(1, a^{2}\right) T C_{22}+1\right)
$$




$$
\begin{aligned}
& I_{2}= \int_{1}^{+\infty}\left[\frac{\left(\ln \left(y+\mathrm{e}^{\delta}\right)\right)^{\delta}}{\left.|\ln | h\right|^{\delta}} \cdot \frac{1}{a^{2} y^{2}}\left|1-\mathrm{e}^{-a^{2} y^{2} t_{1}}\right|+\max \left(1, a^{2}\right) \frac{\left(\ln \left(y^{2}+\mathrm{e}^{\delta}\right)\right)^{\delta}}{\left.|\ln | h\right|^{\delta}} \cdot \frac{1}{a^{2} y^{2}}\left|1-\mathrm{e}^{-a^{2} y^{2} t_{1}}\right|+\int_{t_{1}}^{t} \mathrm{e}^{-a^{2} y^{2}(t-\tau)} \mathrm{d} \tau\right] \mathrm{d} y \\
&=\left.\frac{1}{\left.a^{2}|\ln | h\right|^{\delta}} \int_{1}^{\infty} \frac{\left(\ln \left(y+\mathrm{e}^{\delta}\right)\right)^{\delta}}{y^{2}}\left|1-\mathrm{e}^{-a^{2} y^{2} t_{1} \mid} \mathrm{d} y+\frac{\max \left(1, a^{2}\right)}{\left.a^{2}|\ln | h\right|^{\delta}} \cdot \int_{1}^{\infty} \frac{\left(\ln \left(y^{2}+\mathrm{e}^{\delta}\right)\right)^{\delta}}{y^{2}}\right| 1-\mathrm{e}^{-a^{2} y^{2} t_{1}}\left|\mathrm{~d} y+\int_{1}^{\infty}\right| \int_{t_{1}}^{t} \mathrm{e}^{-a^{2} y^{2}(t-\tau)} \mathrm{d} \tau\right) \mathrm{d} y \\
&= \frac{1}{\left.a^{2}|\ln | h\right|^{\delta}} I_{21}+\frac{\max \left(1, a^{2}\right)}{\left.a^{2}|\ln | h\right|^{\delta}} I_{22}+I_{23} . \\
& I_{21}=\int_{1}^{\infty} \frac{\left(\ln \left(y+\mathrm{e}^{\delta}\right)\right)^{\delta}}{y^{2}}\left|1-\mathrm{e}^{-a^{2} y^{2} t_{1}}\right| \mathrm{d} y \leq \int_{1}^{\infty} \frac{\left(\ln \left(y+\mathrm{e}^{\delta}\right)\right)^{\delta}}{y^{2}} \mathrm{~d} y=C_{21} . \\
& \quad \int_{1}^{\infty}\left(\int_{t_{1}}^{t} \mathrm{e}^{-a^{2} y^{2}(t-\tau)} \mathrm{d} \tau\right) \mathrm{d} y=\frac{1}{a^{2}} \int_{1}^{+\infty} \frac{1}{y^{2}}\left(1-\mathrm{e}^{-a^{2} y^{2}(t-\tau)}\right) \mathrm{d} y \leq \frac{\left.\ln \left(y^{2}+\mathrm{e}^{\delta}\right)\right)^{\delta}}{y^{2}} \mid 1-\mathrm{e}^{-a^{2} y^{2} t_{1} \mid} \mathrm{d} y \leq \int_{1}^{\infty} \frac{\left(\ln \left(y^{2}+\mathrm{e}^{\delta}\right)\right)^{\delta}}{a^{2}|\ln | h \|^{\delta}} \mathrm{d} y=C_{22} . \\
& y_{1}^{2} \frac{\left(\ln \left(y^{2}+\mathrm{e}^{\delta}\right)\right)^{\delta}}{y^{2}} \mathrm{~d} y=\frac{\max \left(1, a^{2}\right)}{\left.a^{2}|\ln | h\right|^{\delta}} C_{23} .
\end{aligned}
$$

Therefore $I_{2} \leq \frac{1}{\left.a^{2}|\ln | h\right|^{\delta}}\left(C_{21}+\max \left(1, a^{2}\right)\left(C_{22}+C_{23}\right)\right)$. Then for $\delta>1-1 / p$, we have

$$
\left(E\left|u_{a_{n}}^{(0)}(x, t)-u_{a_{n}}^{(0)}\left(x_{1}, t_{1}\right)\right|^{2}\right)^{1 / 2} \leq \frac{C_{0}}{|\ln | h||^{\delta}},
$$

where

$$
C_{0}=\frac{\theta}{\pi}\left(T C_{11}+\max \left(1, a^{2}\right) T C_{22}+1+\frac{1}{a^{2}}\left(C_{21}+\max \left(1, a^{2}\right)\left(C_{22}+C_{23}\right)\right)\right), \quad C_{i j}, i=1,2, j=1,2,3 \text { are some con- }
$$
stants.

Consider

$$
\begin{aligned}
&\left(E\left|u_{a_{n}}^{(1)}(x, t)-u_{a_{n}}^{(1)}\left(x_{1}, t_{1}\right)\right|^{2}\right)^{1 / 2}=\left(E\left|\int_{-a_{n}}^{a_{n}} y \sin y x G(y, t) \mathrm{d} y-\int_{-a_{n}}^{a_{n}} y \sin y x_{1} G\left(y_{1}, t_{1}\right) \mathrm{d} y\right|^{2}\right)^{1 / 2} \\
&=\left(\left.E\left|\int_{-a_{n}}^{a_{n}} y\left[\sin y x G(y, t)-\sin y x_{1} G\left(y_{1}, t_{1}\right)\right] \mathrm{d} y\right|^{2}\right|^{1 / 2}\right. \\
&=\left(E\left|\int_{-a_{n}}^{a_{n}} y\left[\left(\sin y x-\sin y x_{1}\right) G\left(y, t_{1}\right)+\left(G(y, t)-G\left(y, t_{1}\right)\right) \sin y x\right] \mathrm{d} y\right|^{2}\right)^{1 / 2} \\
& \leq \int_{-\infty}^{+\infty} y\left[\left|\sin y x-\sin y x_{1}\right|\left(E\left|G\left(y, t_{1}\right)\right|^{2}\right)^{1 / 2}+\left(E\left|G(y, t)-G\left(y, t_{1}\right)\right|^{2}\right)^{1 / 2}\right] \mathrm{d} y \\
&=\int_{-\infty}^{+\infty}\left[\left|\sin y x-\sin y x_{1}\right|\left(y^{2} E\left|G\left(y, t_{1}\right)\right|^{2}\right)^{1 / 2}+\left(y^{2} E \mid G(y, t)-G\left(y, t_{1}\right)^{2}\right)^{1 / 2}\right] \mathrm{d} y . \\
&\left(y^{2}\left|G\left(y, t_{1}\right)\right|^{2}\right)^{1 / 2} \leq \frac{1}{\sqrt{2 \pi}} \int_{0}^{t_{1}} \mathrm{e}^{-a^{2} y^{2}(t-\tau)}\left(y^{2} E|\tilde{\xi}(y, \tau)|^{2}\right)^{1 / 2} \mathrm{~d} \tau .
\end{aligned}
$$

From Lemma 4, we obtain 


$$
\begin{aligned}
\left(y^{2} E|\xi(y, \tau)|^{2}\right)^{1 / 2} & =\frac{1}{\sqrt{2 \pi}}\left(E\left|\int_{-\infty}^{+\infty} y \cos y x \xi(x, \tau) \mathrm{d} x\right|^{2}\right)^{1 / 2} \\
& \leq \frac{1}{\sqrt{2 \pi}}\left(E\left|\int_{-\infty}^{+\infty} \sin y x \frac{\partial \xi(x, \tau)}{\partial x} \mathrm{~d} x\right|^{2}\right)^{1 / 2} \\
& \leq \frac{1}{\sqrt{2 \pi}} \int_{-\infty}^{+\infty}\left(E\left|\frac{\partial \xi(x, \tau)}{\partial x}\right|^{2}\right)^{1 / 2} \mathrm{~d} x<\frac{1}{\sqrt{2 \pi}} \theta_{1} .
\end{aligned}
$$

Similarly $\left(E\left|u_{a_{n}}^{(1)}(x, t)-u_{a_{n}}^{(1)}\left(x_{1}, t_{1}\right)\right|^{2}\right)^{1 / 2} \leq \frac{C_{1}}{\left.|\ln | h\right|^{\delta}}$

where $C_{1}=\frac{\theta}{\pi}\left(T C_{11}+\max \left(1, a^{2}\right) T C_{22}+1+\frac{1}{a^{2}}\left(C_{21}+\max \left(1, a^{2}\right)\left(C_{22}+C_{23}\right)\right)\right), \quad C_{i j}, i=1,2, j=1,2,3$ are some constants.

Consider

$$
\begin{aligned}
& \left(E\left|u_{a_{n}}^{(2)}(x, t)-u_{a_{n}}^{(2)}\left(x_{1}, t_{1}\right)\right|^{2}\right)^{1 / 2} \\
= & \left(\left.E\left|\int_{-a_{n}}^{a_{n}} y^{2} \cos y x G(y, t) \mathrm{d} y-\int_{-a_{n}}^{a_{n}} y^{2} \cos y x_{1} G\left(y, t_{1}\right) \mathrm{d} y\right|^{2}\right|^{1 / 2}\right. \\
= & \left(\left.\left.E\left|\int_{-a_{n}}^{a_{n}} y^{2}\left[\cos y x G(y, t)-\cos y x_{1} G\left(y, t_{1}\right)\right] \mathrm{d} y\right|^{2}\right|^{1 / 2}\right|^{1 / 2}\right. \\
= & \left(E\left|\int_{-a_{n}}^{a_{n}} y^{2}\left[\left(\cos y x-\cos y x_{1}\right) G\left(y, t_{1}\right)+\left(G(y, t)-G\left(y, t_{1}\right)\right) \cos y x\right] \mathrm{d} y\right|^{2}\right)^{1 / 2} \\
\leq & \int_{-\infty}^{+\infty} y^{2}\left[\left|\cos y x-\cos y x_{1}\right|\left(E\left|G\left(y, t_{1}\right)\right|^{2}\right)^{1 / 2}+\left(E\left|G(y, t)-G\left(y, t_{1}\right)\right|^{2}\right)^{1 / 2}\right] \mathrm{d} y \\
= & \int_{-\infty}^{+\infty}\left[\left|\cos y x-\cos y x_{1}\right|\left(y^{4} E\left|G\left(y, t_{1}\right)\right|^{2}\right)^{1 / 2}+\left(y^{4} E\left|G(y, t)-G\left(y, t_{1}\right)\right|^{2}\right)^{1 / 2}\right] \mathrm{d} y . \\
& \left(y^{4}\left|G\left(y, t_{1}\right)\right|^{2}\right)^{1 / 2} \leq \frac{1}{\sqrt{2 \pi}} \int_{0}^{t_{1}} \mathrm{e}^{-a^{2} y^{2}(t-\tau)}\left(y^{4} E|\tilde{\xi}(y, \tau)|^{2}\right)^{1 / 2} \mathrm{~d} \tau .
\end{aligned}
$$

From Lemma 4 we obtain

$$
\begin{aligned}
\left(y^{4} E|\xi(y, \tau)|^{2}\right)^{1 / 2} & =\frac{1}{\sqrt{2 \pi}}\left(E\left|\int_{-\infty}^{+\infty} y^{2} \cos y x \xi(x, \tau) \mathrm{d} x\right|^{2}\right)^{1 / 2} \\
& \leq \frac{1}{\sqrt{2 \pi}}\left(E\left|\int_{-\infty}^{+\infty} \cos y x \frac{\partial^{2} \xi(x, \tau)}{\partial x^{2}} \mathrm{~d} x\right|^{2}\right)^{1 / 2} \\
& \leq \frac{1}{\sqrt{2 \pi}} \int_{-\infty}^{+\infty}\left(E\left|\frac{\partial^{2} \xi(x, \tau)}{\partial x^{2}}\right|^{2}\right)^{1 / 2} \mathrm{~d} x<\frac{1}{\sqrt{2 \pi}} \theta_{2} .
\end{aligned}
$$


Then $\left(E\left|u_{a_{n}}^{(2)}(x, t)-u_{a_{n}}^{(2)}\left(x_{1}, t_{1}\right)\right|^{2}\right)^{1 / 2} \leq \frac{C_{2}}{\left.|\ln | h\right|^{\delta}}$,

where $C_{2}=\frac{\theta_{2}}{\pi}\left(T C_{11}+\max \left(1, a^{2}\right) T C_{22}+1+\frac{1}{a^{2}}\left(C_{21}+\max \left(1, a^{2}\right)\left(C_{22}+C_{23}\right)\right)\right) \quad C_{i j}, i=1,2, j=1,2,3$ are some constants.

\section{Estimates of the Distribution of the Supremum of a Solution}

Theorem 8. [9] Let $R^{k}$ be the $k$-dimensional space, $\mathrm{d}(t, s)=\max _{1 \leq i \leq k}\left|t_{i}-s_{1}\right|, \quad T=\left\{0 \leq t_{i} \leq T_{i}, n=1,2, \cdots, k\right\}$, $T_{i}>0$. Assume that $X=\{X(t), t T\}$ is separable and $X \in \operatorname{Sub}_{\varphi}(\Omega)$. If $\sup _{\mathrm{d}(t, s) \leq h} \tau_{\phi}(X(t)-X(s)) \leq \sigma(h)$, where $\sigma(h)$ is a monotone increasing continuous function such that $\sigma(h) \rightarrow 0$ as $h \rightarrow \infty$, and $\int_{0+} \psi\left(\ln \frac{1}{\sigma^{(-1)}(\varepsilon)}\right) \mathrm{d} \varepsilon<\infty$, where $\psi(u)=u / \phi^{(-1)}(u)$ and $\sigma^{(-1)}(\varepsilon)$ is the inverse function to $\sigma(\varepsilon)$. Then $P\left\{\sup _{t \in T}|X(t)|>u\right\} \leq 2 \tilde{A}(u, \theta)$, for all $0<\theta<1$ and $u>\frac{2 I_{\varphi}\left(\theta \varepsilon_{0}\right)}{\theta(1-\theta)}$, where

$$
\begin{gathered}
\tilde{A}(u, \theta)=\exp \left\{-\varphi^{*}\left(\frac{1}{\varepsilon_{0}}\left[u(1-\theta)-\frac{2}{\theta} \tilde{I}_{\phi}\left(\theta \tilde{\varepsilon}_{0}\right)\right]\right)\right\}, \\
\tilde{\varepsilon}_{0}=\sup _{t \in T}\left(E|X(t)|^{2}\right)^{1 / 2} \\
\tilde{I}_{\varphi}(\delta)=\int_{0}^{\delta} \psi\left(\sum_{i=1}^{k} \ln \left(\frac{T_{i}}{2 \sigma^{(-1)}(\varepsilon)}+1\right)\right) \mathrm{d} \varepsilon .
\end{gathered}
$$

Theorem 9. Let the conditions of Theorem 6 hold

$$
u(x, t)=\int_{-\infty}^{+\infty} \cos y x G(y, t) \mathrm{d} y .
$$

where

$$
\begin{gathered}
G(y, t)=\frac{1}{\sqrt{2 \pi}} \int_{0}^{t} \mathrm{e}^{-a^{2} y^{2}(t-\tau)} \tilde{\xi}(y, \tau) \mathrm{d} \tau, \\
\tilde{\xi}(y, \tau)=\frac{1}{\sqrt{2 \pi}} \int_{-\infty}^{+\infty} \cos y x \xi(x, \tau) \mathrm{d} x \\
(x, t) \in D, D=[-A, A] \times[0, T] \text {. Then } \\
P\left\{\sup _{(x, t) \in D}|u(x, t)|>u\right\} \leq 2 \tilde{A}(u, \theta),
\end{gathered}
$$

for all $0<\theta<1$ and $u>\frac{2 I_{\varphi}\left(\theta \tilde{\varepsilon}_{0}\right)}{\theta(1-\theta)}$, where

$$
\tilde{A}(u, \theta)=\exp \left\{-\varphi^{*}\left(\frac{1}{\varepsilon_{0}}\left[u(1-\theta)-\frac{2}{\theta} \tilde{I}_{\varphi}\left(\theta \tilde{\varepsilon}_{0}\right)\right]\right)\right\},
$$




$$
\begin{gathered}
\tilde{\varepsilon}_{0}=\sup _{(x, t) \in D}\left(E|u(x, t)|^{2}\right)^{1 / 2}, \\
\tilde{I}_{\phi}\left(\theta \tilde{\varepsilon}_{0}\right)=\int_{0}^{\theta \tilde{\varepsilon}_{0}} \psi\left(\ln \left(\frac{T}{2 \sigma^{(-1)}(\varepsilon)}+1\right)+\ln \left(\frac{A}{\sigma^{(-1)}(\varepsilon)}+1\right)\right) \mathrm{d} \varepsilon,
\end{gathered}
$$

where $\sigma(\varepsilon)$ is a monotone increasing continuous function such that $\sigma(\varepsilon) \rightarrow 0$ as $\varepsilon \rightarrow 0$, and $\sigma^{(-1)}(\varepsilon)$ is the inverse function to $\sigma(\varepsilon)$.

Proof. This theorem follows from Theorem 8.

\section{References}

[1] de Feriet, K. (1962) Statistical Mechanics of Continuous Media. Proceedings of Symposia in Applied Mathematics, American Mathematical Society, Providence, 165-198.

[2] Beisenbaev, E. and Kozachenko, Yu.V. (1979) Uniform Convergence in Probability of Random Series, and Solutions of Boundary Value Problems with Random Initial Conditions. Theory of Probability and Mathematical Statistics, 21, 9-23.

[3] Buldygin, V.V. and Kozachenko, Yu.V. (1979) On a Question of the Applicability of the Fourier Method for Solving Problems with Random Boundary Conditions. Random Processes in Problems Mathematical Physics, Academy of Sciences of Ukrain.SSR, Institute of Mathematics, Kuiv, 4-35.

[4] de La Krus, E.B. and Kozachenko, Yu.V. (1995) Boundary-Value Problems for Equations of Mathematical Physics with Strictly Orlics Random Initial Conditios. Random Operators and Stochastic Equations, 3, 201-220. http://dx.doi.org/10.1515/rose.1995.3.3.201

[5] Kozachenko, Yu.V. and Endzhyrgly (1994) Justification of Applicability of the Fourier Method to the Boundary-Value Problems with Random Initial Conditions I. Theory of Probability and Mathematical Statistics, 51, 78-89.

[6] Kozachenko, Yu.V. and Endzhyrgly (1994) Justification of Applicability of the Fourier Method to the Boundary-Value Problems with Random Initial Conditions II. Theory of Probability and Mathematical Statistics, 53, 58-68.

[7] Kozachenko, Yu.V. and Kovalchuk, Ya.A. (1998) Boundary Value Problems with Random Initial Conditions and Series of Functions of $\operatorname{Sub}_{\varphi}(\Omega)$. Ukrainian Mathematical Journal, 50, 504-515.

[8] Dovgay, B.V., Kozachenko, Yu.V. and Slyvka-Tylyshchak, G.I. (2008) The Boundary-Value Problems of Mathematical Physics with Random Factors. Kyiv University, Kyiv, 173 p. (Ukrainian)

[9] Kozachenko, Yu.V. and Slyvka, G.I. (2004) Justification of the Fourier Method for Hyperbolic Equations with Random Initial Conditions. Theory of Probability and Mathematical Statistics, 69, 67-83. http://dx.doi.org/10.1090/S0094-9000-05-00615-0

[10] Slyvka, A.I. (2002) A Boundary-Value Problem of the Mathematical Physics with Random Initials Conditions. Bulletin of University of Kyiv. Series: Physics \& Mathematics, 5, 172-178.

[11] Slyvka-Tylyshchak, A.I. (2012) Justification of the Fourier Method for Equations of Homogeneous String Vibration with Random Initial Conditions. Annales Universitatis Scientarium Budapestinensis de Rolando Eötvös Nominatae Sectio Mathematica, 38, 211-232.

[12] Kozachenko, Y.V. and Slyvka, G.I. (2007) Modelling a Solution of a Hyperbolic Equation with Random Initial Conditions. Theory Probability and Mathematical Statistics, 74, 59-75.

[13] Tylyshchak, A.I.S. (2012) Simulation of Vibrations of a Rectangular Membrane with Random Initial Conditions. Annales Mathematicae and Informaticae, 39, 325-338.

[14] Dovgay, B.V. and Kozachenko, Y.V. (2005) The Condition for Application of Fourie Method to the Solution of Nongomogeneous String Oscillation Equation with $\varphi$-Subgaussianright Hand Side. Random Operators and Stochastic Equations, 13, 281-296.

[15] Kozachenko, Y.V. and Veresh, K.J. (2010) The Heat Equation with Random Initial Conditions from Orlicz Space. Theory of Probability and Mathematical Statistics, 80, 71-84. http://dx.doi.org/10.1090/S0094-9000-2010-00795-2

[16] Kozachenko, Y.V. and Veresh, K.J. (2010) Boundary-Value Problems for a Nonhomogeneous Parabolic Equation with Orlicz Right Side. Random Operators and Stochastic Equations, 18, 97-119. http://dx.doi.org/10.1515/rose.2010.005

[17] Angulo, J.M., Ruiz-Medina, M.D., Anh, V.V. and Grecksch, W. (2000) Fractional Diffusion and Fractional Heat Equation. Advances in Applied Probability, 32, 1077-1099. http://dx.doi.org/10.1239/aap/1013540349

[18] Kozachenko, Y.V. and Leonenko, G.M. (2006) Extremal Behavior of the Heat Random Field. Extremes, 8, $191-205$. http://dx.doi.org/10.1007/s10687-006-7967-8 
[19] Beghin, L., Kozachenko, Y., Orsingher, E. and Sakhno, L. (2007) On the Solution of Linear Odd-Order Heat-Type Equations with Random Initial. Journal of Statistical Physics, 127, 721-739. http://dx.doi.org/10.1007/s10955-007-9309-X

[20] Ratanov N.E., Shuhov, A.G. and Suhov, Y.M. (1991) Stabilization of the Statistical Solution of the Parabolic Equation. Acta Applicandae Mathematicae, 22, 103-115.

[21] Buldygin, V.V. and Kozachenko, Y.V. (2000) Metric Characterization of Random Variables and Random Processes. American Mathematical Society, Rhode.

[22] Antonini, R.G., Kozachenko, Y. and Nikitina, T. (2003) Spaces of $\varphi$-Subgaussian Random Variables. Memorie di Matematica e Applicazioni, Accademia Nazionale delle Scinze detta dei XL, Vol. 27, 95-124.

[23] Krasnoselsky, M.A. and Rutitcky, Y.B. (1961) Convex Functions and Orlicz Spaces. Noordhof, Gröningen.

[24] Kozachenko, Y.V. and Ostrovskij, E.V. (1986) Banach Spaces of Random Variables of Sub-Gaussian Type. Theory of Probability and Mathematical Statistics, 532, 42-53.

[25] Markovich, B.M. (2010) Equations of Mathematical Physics. Lviv Polytechnic Publishing House, Lviv, 384 p. (Ukrainian).

[26] Budylin, A.M. (2002) Fourier Series and Integrals. Saint Petersburg, 137 p. 
Scientific Research Publishing (SCIRP) is one of the largest Open Access journal publishers. It is currently publishing more than 200 open access, online, peer-reviewed journals covering a wide range of academic disciplines. SCIRP serves the worldwide academic communities and contributes to the progress and application of science with its publication.

Other selected journals from SCIRP are listed as below. Submit your manuscript to us via either submit@scirp.org or Online Submission Portal.
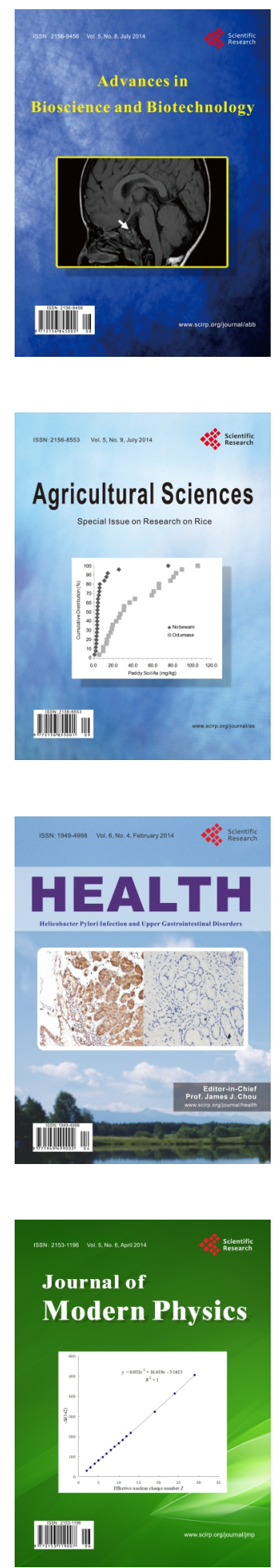
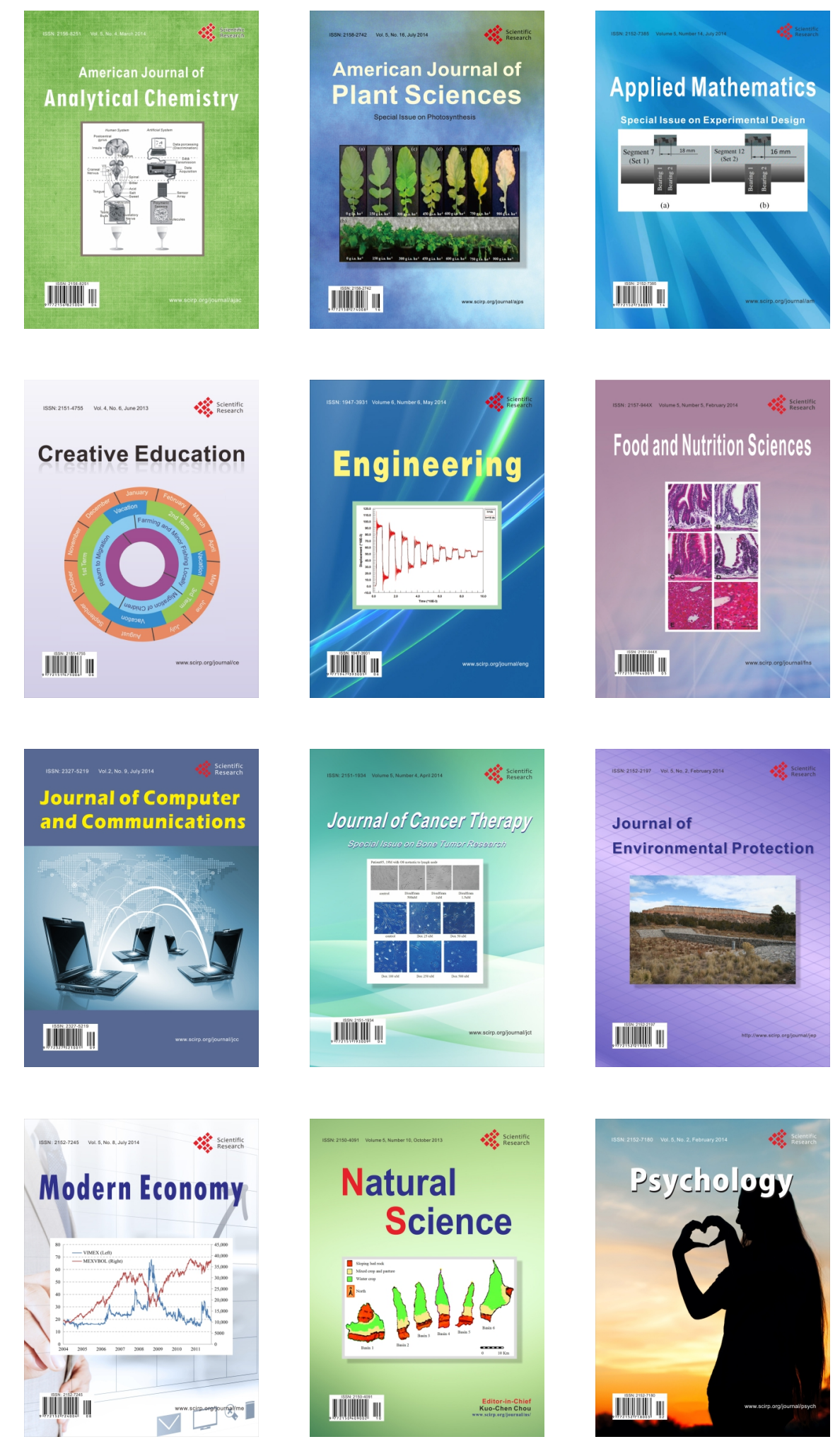